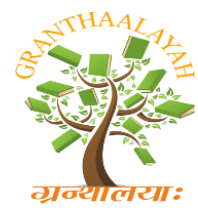

INTERNATIONAL JOURNAL OF RESEARCH -

GRANTHAALAYAH

A knowledge Repository

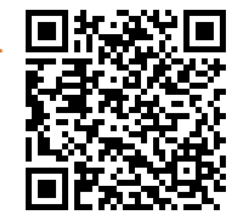

Science

\title{
GEOMETRY MODELING OF 3D TURBINE STAGES - HOW TO OBTAIN HIGH QUALITY GRID AND OVERCOME DISCRETIZATION PROBLEMS
}

\author{
Galina Ilieva *1 \\ ${ }^{* 1}$ Centre for Mechanical and Aerospace Science and Technology, University of Beira Interior, \\ Covilhã, PORTUGAL
}

\begin{abstract}
The process of geometry modeling of $3 D$ turbine blades is basically related to the necessity of finite elements with high quality to be obtained, in the process of flow domain approximation. Various approaches for geometry modeling and grid generation, ways to attain elements of high quality and having positive volumes, have been under research and are presented in current paper. Developed methodology and established techniques to high quality grid, are implemented into practice, for geometry modeling of a wide range of turbine and compressor blades of complex geometry.
\end{abstract}

Keywords:

quality, geometry modeling, negative volume, skewed element, turbine blade.

Cite This Article: Galina Ilieva, "GEOMETRY MODELING OF 3D TURBINE STAGES HOW TO OBTAIN HIGH QUALITY GRID AND OVERCOME DISCRETIZATION PROBLEMS" International Journal of Research - Granthaalayah, Vol. 4, No. 2 (2016): 197-205.

\section{INTRODUCTION}

Modeling, research and analysis of aerodynamic performance of 3D compressible, viscous and turbulent flow, through complex stages, requires fulfillment of geometry and its high quality mesh, in order to obtain physically correct prediction of fluid parameters distribution. In a comparison with many software products, for geometry modeling, Gambit-Turbo permits the user to construct turbo geometries in a faster way. However, instead of all implemented advanced options, the mesh procedure can result in a grid with left-handed elements, low quality elements, negative volumes and others, that are a prerequisite to physically incorrect results for flow parameters distribution or lead to solution divergence. The accuracy of a CFD solution depends on the achieved grid quality.

This study is targeted to development, verification and implementation of a methodology for geometry modeling of 3D turbine cascades of complex geometry, discretized with high quality 
elements, obtained after application of a set of various logical procedures, developed to eliminate the presence of bad elements and solve all mesh problems and challenges.

\section{PROBLEM SET UP}

A 3D turbine cascade with twisted rotor blade is under consideration. The 3D model is built in Gambit, after implementation of a logical sequence and specific approaches to meet requirements for high quality grid.

It is very important, for all users, to understand that both logic and order in which are constructed all lines, surfaces, sub-domains and volumes, is crucial for the upcoming mesh procedures, grid consistency and obtained 2D or 3D elements with very high quality.

The following sequence is advisable for 3D modeling of stator and rotor blades:

1. Introduction of the key points' coordinates to construct hub and shroud sections for stator and rotor blades as is discussed in $[1,2]$.

2. Leading, trailing and side contours set-up; definition of inlet and outlet geometry angles on the basis of already built basic contours and blade tip vertices. It is found that the order, in which the blade tip vertices are selected, is crucial in the process of the turbo profile definition. The blade tip vertices must be selected in an order going from hub to shroud sections.

3. Realization of stator and rotor sub-volumes, which build the stage main volumes. Definition of main turbo zones: hub, casing, pressure and suction side domains. All required turbo volume characteristics are determined by the turbo profile and by specification of number of blades, tip clearance (if any), and number of span-wise sections.

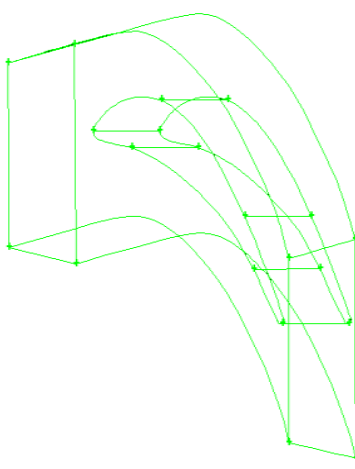

(a)

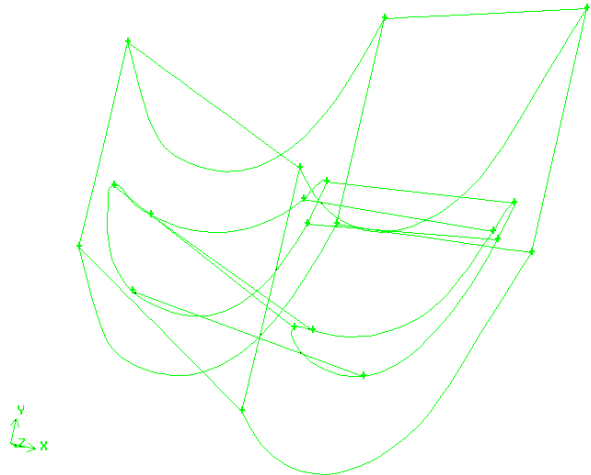

(b)

Figure1 ( $\boldsymbol{a}, \boldsymbol{b})$ : Volumes formed around stator (a) and rotor blades.

4. Boundary layer creation. In this step of the geometry modeling procedure, is of a great importance to create boundary layer, that have enough thickness, distributed in several rows of elements, by application of the appropriate "growing factor" value.

Sizing functions could be applied effectively to define the size of boundary cells. However, they can't contribute to the required directional control (anisotropy), so one possible approach is to build elements from the boundaries and to form "viscous" layers, fig. 3. 
It is clear that velocity and often temperature gradients, in a direction normal to the streamed walls, are much larger than the gradients parallel to the wall. This is the reason to implement "inflation layer control". It creates thin elements that can capture the normal gradient with minimal elements; use inflation layers to correctly capture the velocity and temperature gradients near the no-slip walls.

5. Discretization of hub section, next is application of Cooper's scheme to create needed 3D grid around turbine blades, fig. 2(a,b). Methods to attain high quality 3D elements, with positive volumes were implemented [2,3].

6. Elements quality check, fig.4 (a,b).

7. Definition of boundary zones, in this case: "wall", "pressure-inlet", "pressure-outlet", "fluid" "periodic", fig. 5 (a,b).

8. Merge of stator and rotor main volumes, fig.6.

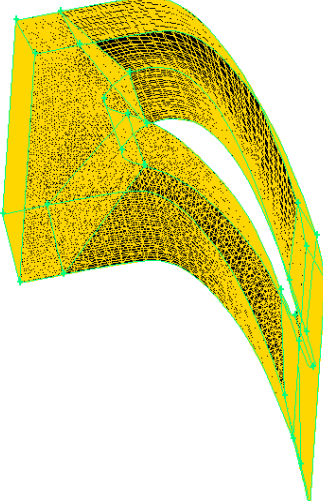

(a)

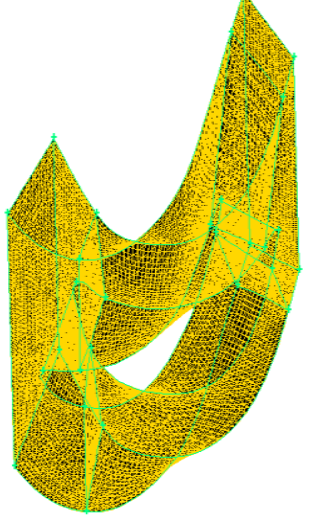

(b)

Figure $2(\boldsymbol{a}, \boldsymbol{b})$ : Discretization of stator (a) and rotor (b) blade volumes.

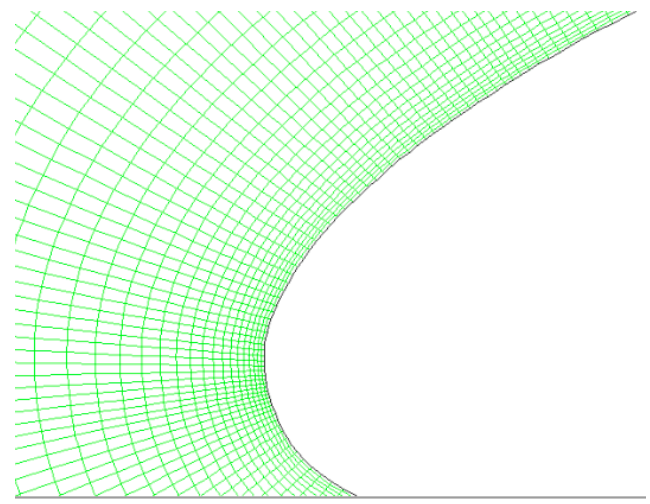

Figure 3: Viscous layer around the leading edge of the stator blade. 


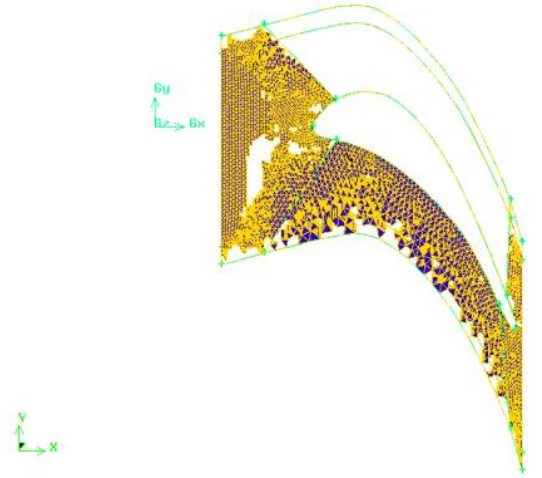

(a)

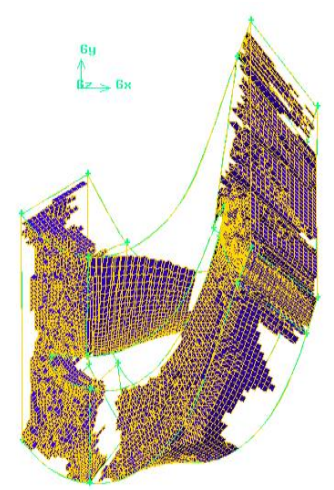

(b)

Figure $4(\boldsymbol{a}, \boldsymbol{b})$ : Element quality check for stator (a) and rotor (b) volumes.

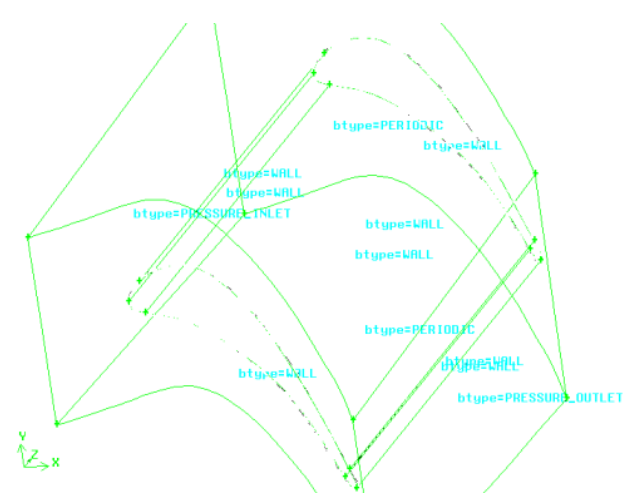

(a)

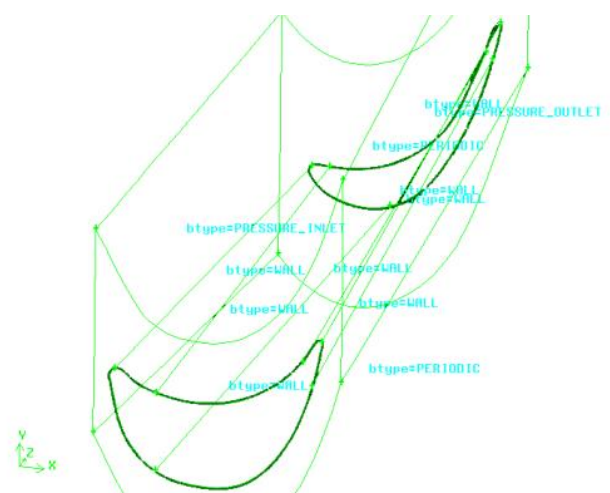

(b)

Figure $5(\boldsymbol{a}, \boldsymbol{b})$ : Defined boundary zones for stator (a) and rotor (b) turbo volume.

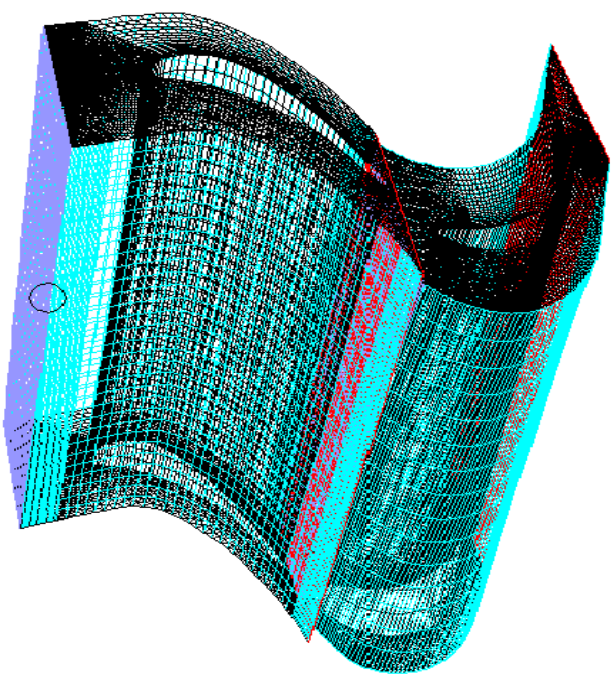

Figure 6: Merged stator and rotor volumes. 


\section{SPECIFIC FEATURES, PROBLEMS AND SOLUTIONS}

1. Discretization of turbo volumes with grid consisted of high quality elements is very important in the geometry modeling. Gambit gives opportunity for grid quality check. Level of "equiangular skew" for grid elements, in the range of 0 to 1, and presence of negative volumes can be determined. The highest advisable value for 3D grid elements quality check is between 0,6-0,7 [1]. Presence of quality over 0.7 in the resulting mesh can greatly affect the accuracy and robustness of the CFD solution [1,3]. However, in current research is found that advisable value of quality factor is $0.3-0.5$. A value of 0.7 can provide elements with level of skewness, leading to some solution instabilities, especially in case of vortex structures, boundary layer separation, unsteady effects, among others to appear in the flow $[2,3]$.

As a result of the extensive research works, it is found that the high level of skewness in downstream direction is a reason for large changes of the unknown parameters (jump in gradients) and robust iterative procedure. Bad elements (elements of low quality) could be avoided by: mesh refinement around already existing nodes and/or elements; re-mesh in the problematic areas; application of "double sided" mesh option; change in values for "internal ratio" and other options; change of elements type and/or change in the chosen scheme for flow mesh procedures. Other established approaches and many examples of solved serious problems are discussed in details in [3].

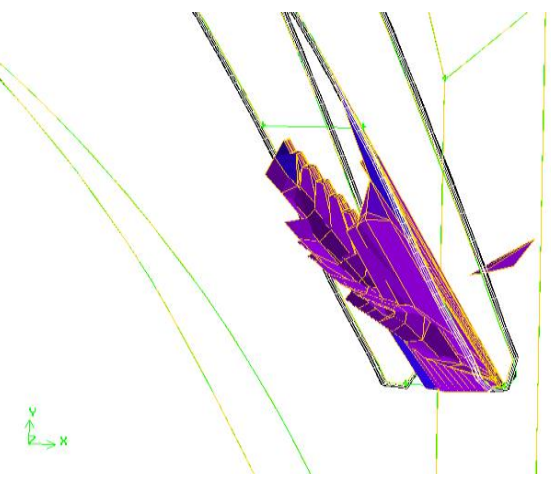

(a)

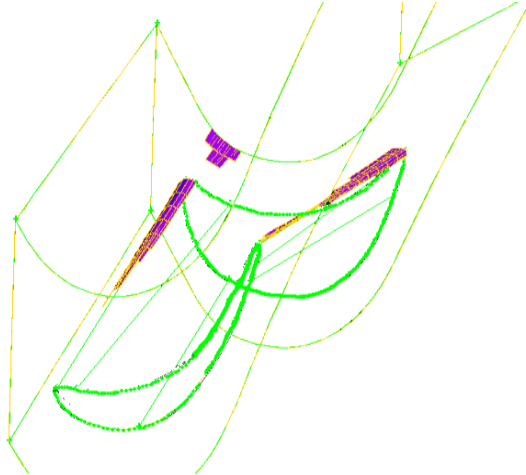

(b)

Figure $7(\boldsymbol{a}, \boldsymbol{b})$ : Elements with negative volume, formed at stator trailing edge (a) and at rotor leading and trailing edges (b), in radial direction.

2. It is found that, normally, zones with low quality elements, negative volumes and lefthandedness, especially in case of applied Cooper's discretization scheme, are concentrated close to the leading and trailing edges, in radial direction. This is a problem provoked of the blade twistiness, fig.7 a,b.

For example, current studies show that the increase of "internal ratio" values, for those lines describing the leading and trailing edges, and the application of Cooper's scheme, for flow discretization, lead to increase in the number of negative volumes. Research works demonstrate the presence of overlapping elements, in radial direction. It comes as a result of inappropriate choice for mesh factors and their values, applied in the process of finite element areas coping, from hub section to shroud section, in case Cooper's method for 3D discretization is applied. 
It is proven that an optimal value for "internal count" option to dicretize each line, describing profile contours must be set up. What will be that value depends on many factors such as blade's size, consistency of the boundary layer elements and chosen scheme for its creation, curvature of blade walls, etc.

Number of finite elements, used for approximation of leading, trailing and side lines of blade sections, along the blade height, has to be defined very precisely. Profile contours could be resolved with high quality elements by activation of the "successive ratio" option, from "Mesh Edges" panel, for grading those nodes along the leading and trailing edges, fig.8 (a,b). In fig. 9(a) is shown problematic mesh at the trailing edge of the rotor blade of hub section; fig 9(b) presents the resulting negative elements, along the blade height, after application of Cooper's scheme for discretization.

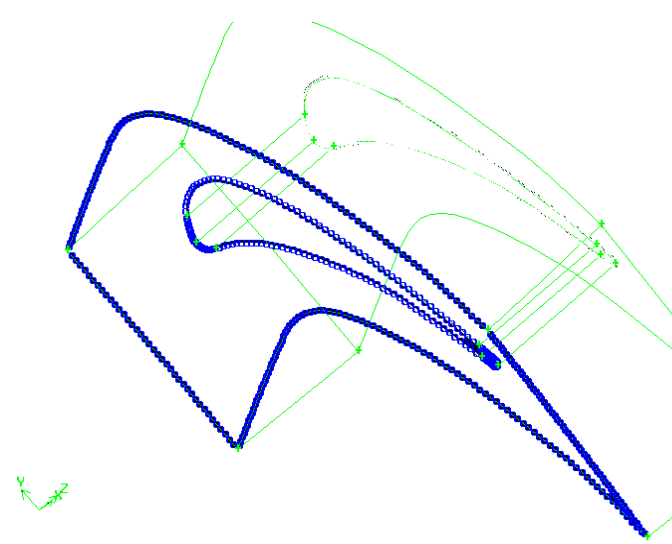

(a)

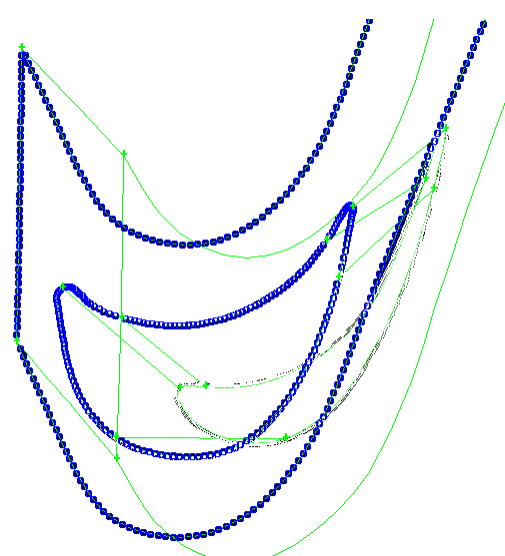

(b)

Figure $8(\boldsymbol{a}, \boldsymbol{b})$ : Approximation of leading, trailing and sideward edges of stator (a) and rotor (b) blades; sections at hub of blades under consideration.

Research clearly shows that for grading of nodes, distributed along the trailing and leading edges (close to the end vertices of the central camber line), value of the "successive ratio" option, must be significantly increased. It's advisable, in the process of turbo volume discretization, to define higher value for "internal size" option, in order to escape presence of skewed elements in radial direction, fig. 10.

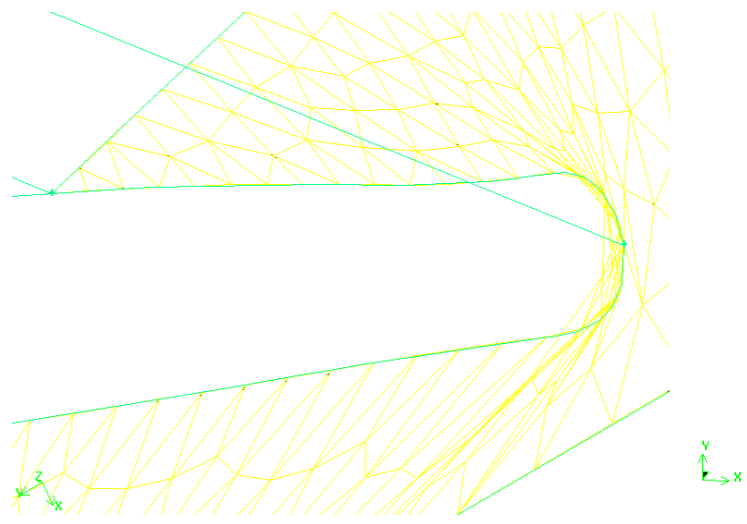

(a)

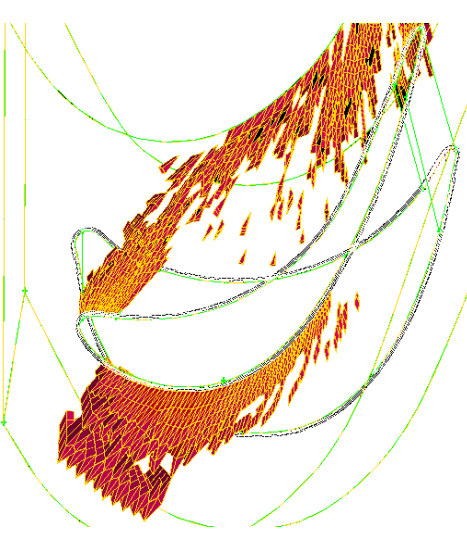

(b)

Figure $9(\boldsymbol{a}, \boldsymbol{b})$ : Highly skewed elements for stator (a) and rotor blades (b). 
Problems related to presence of negative volumes couldn't be eliminated after application of one or another approach, a complex method to overcome all discussed problems must be implemented: modification of already applied discretization schemes; mutually connected values for "internal size" and "successive ratio" options; appropriate type and number of finite elements, which approximate stator and rotor volumes, etc.

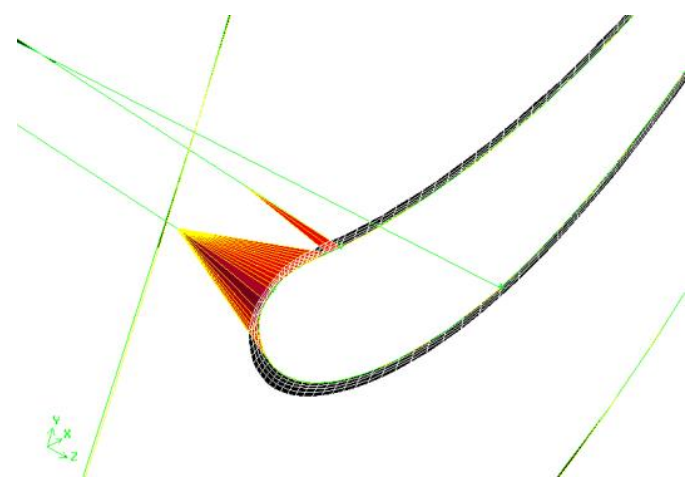

Figure 10: Highly skewed elements in radial direction.

For different blade geometries, various individual approaches to attain high quality grid, are available. For instance, flow domain approximation, is very sensible of the blade geometry. For leading and trailing edges, the presence of highly skewed elements is a prerequisite to worse mesh quality, fig.11. In this case, bad quality elements can lower with increase of discretization level along the foil contours. The ttendency is to gain a grid with good level of commensurability for edges. It could be achieved after a detailed grid analyses.

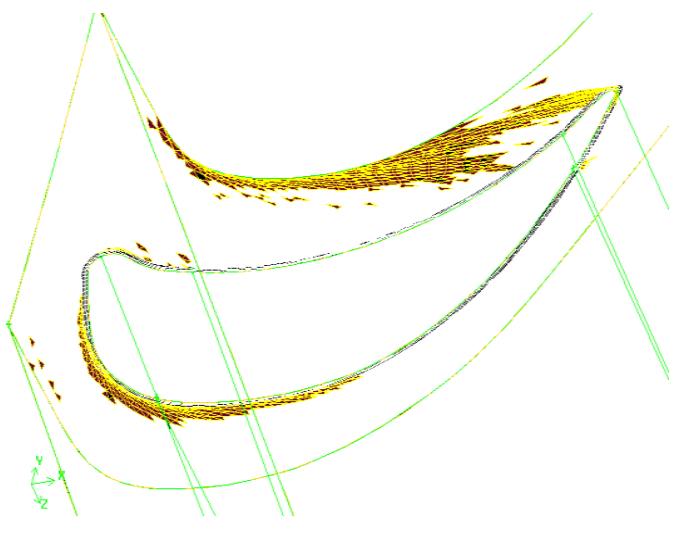

Figure 11: Highly skewed elements in hub section of rotor blade.

As a result of extensive research works, the following approach to escape from presence of skewed elements, formed along hub and shroud sections, is advisable:

3. individual discretization of lines, describing hub and shroud sections with appropriate values for "internal size" and "successive ratio" options;

4. discretization of hub section after applying appropriate set of options by type and value;

5. quality check of the resulting mesh;

6. discretization of volumes; 
7. Quality check of the volume elements.

8. To obtain high quality grid, the default values for "grading" and "max faces", must be changed in agreement with the case under research and according to conformities that were found.

9. Before the *.msh file is read in Fluent, boundary types to all turbo domains, must be specified. Boundary zones are of the type: "wall", "pressure-inlet", "pressure-outlet", "periodic", in this case of research.

10. For correct definition of periodic boundary zones, sideward faces must be selected in precise sequence and meshed in a "linked" mode. This leads to correct sign and value of periodic boundaries that are defined of rotational type.

11. Merge of turbo volumes after their geometry modeling is finalized.

\section{CONCLUSIONS \& RECOMMENDATIONS}

On the basis of the performed research works, a methodology for 3D geometry modeling of turbine stages is established. The methodology provides high quality grid, following mentioned in brief in next lines recommendations.

Cooper's discretization scheme for fluid domain approximation of twisted rotor blade is required. Cooper's scheme is following the blade shape, in radial direction, in the process of volume grid generation.

For mesh refinement, close to both leading and trailing edges, discretization for each line of profile contours must be carried out, at definite values for the mesh options. Resulting mesh must be consistent with shape and size of elements that are building the boundary layer. It's important to acquire elements with commensurable edge lengths along foil section and boundary layer. All this is a prerequisite to overcome the presence of highly skewed elements when transfer from one to another section along the blade height.

Values of "successive ratio" option, when approximating the blade contours, close to the camber line end points, radial direction, must be carefully determined.

Values for "internal count" and "successive ratio" options, must be commensurable, especially for leading and trailing edges. Very low values, for both options, lead to creation of negative elements when Cooper's scheme is used for volume discretization.

As a result of fulfilled research works, is found that the increase of grading option, for nodes along leading and trailing edges, and the decrease of "internal count" value, lead to high skewness and presence of negative volumes close to the shroud section, when Cooper's scheme is applied for approximation.

In definite cases the decomposition approach results into boundary layer elements of low quality distributed along the pressure and suction sides. 
A methodology and approaches for high quality mesh grid is achieved [2, 3]. Both the developed methodology and approaches are implemented into practice for geometry modeling of a wide range of turbine blades.

\section{ACKNOWLEDGEMENTS}

Acknowledgements to prof. Rumen Yossifov for encouraging me during my first steps in GAMBIT and FLUENT years ago.

\section{REFERENCES}

[1] Ansys - Fluent: GAMBIT User's http://aerojet.engr.ucdavis.edu/gambithelp/html/users_guide/ugtoc.htm

[2] Galina Ilieva, "Modeling, Research andAnalisys of 3D Real Flow in Turbine Stages with Complex Geometry", Thesis, TU-Varna.

[3] Galina Ilieva, Numerical Modeling and Research of 3D Turbine Stage, Engineering Applications of Computational Fluid Dynamics, Springer, Advanced Structured Materials series, 2015, Vol.44, pp.103-126, DOI 10.1007/978-3-319-02836-1_8, Online ISBN978-3-319-02836-1. 